\title{
SINTOMÁTICOS RESPIRATORIOS EN PERSONAS ATENDIDAS EN ESTABLECIMIENTOS DE SALUD DEL MINISTERIO DE SALUD EN LIMA, PERÚ
}

\author{
Lely Solari ${ }^{1,2, a}$, Jorge O. Alarcón ${ }^{3, b}$, Julia Piscoya ${ }^{3, c}$, Romina Tejada $^{1, c}$
}

\begin{abstract}
RESUMEN
Objetivo. El objetivo de este estudio fue determinar el porcentaje de sintomáticos respiratorios (SR), según la definición programática, entre las personas que en condiciones habituales acuden a consulta en los establecimientos de salud de primer nivel del Ministerio de Salud en Lima, e identificar el porcentaje de pacientes con tuberculosis entre los SR y entre tosedores de más de siete días. Materiales y métodos. Estudio transversal en pacientes que acudieron a consulta en 57 centros de salud. Se identificó a los pacientes con tos y expectoración de más de siete días y SR, a quienes se les tomó una muestra de esputo para descarte de tuberculosis mediante baciloscopía y cultivos. Resultados. Se encuestaron 10421 personas. El 2,7\% presentaron tos con expectoración por $\geq 7$ días y solo el 1,1\% fueron SR; 215 pacientes fueron examinados para descarte de tuberculosis. Siete $(5,9 \%)$ de los SR y ocho $(4,8 \%)$ de los pacientes con tos de 7-14 días tuvieron tuberculosis. Conclusión. El porcentaje de SR en establecimientos de salud del Ministerio de Salud en Lima fue menor al $5 \%$ esperado, con un rango de $0,8 \%$ en Callao hasta 1,5\% en Lima Ciudad. El porcentaje de tuberculosis entre tosedores de 7-14 días y SR concuerda con el hallado en otros estudios locales. Se debe considerar la posibilidad de disminuir la meta de SR a menos del $5 \%$ y ampliar el despistaje de tuberculosis a pacientes con tos $\geq 7$ días en las Direcciones de Salud con altas incidencias de tuberculosis.
\end{abstract}

Palabras clave: Cribado; Tuberculosis; Sistemas de Salud; Perú (fuente: DeCS BIREME).

\section{RESPIRATORY SYMPTOMS IN PEOPLE ATTENDED IN HEALTH FACILITIES OF THE MINISTRY OF HEALTH IN LIMA, PERU}

\begin{abstract}
Objective. The aim of this study was to determine the percentage of symptomatic respiratory (SR) cases, according to the programmatic definition, among people who attend medical offices at first-level health facilities of the Ministry of Health in Lima under normal circumstances. The study also aims at identifying the percentage of patients with tuberculosis among the RS and among coughers over seven days. Materials and Methods. Cross-sectional study of patients attending consultations at 57 health centers. Patients with cough and phlegm lasting more than seven days and RS were identified and a sputum sample was taken to rule out tuberculosis by smear and culture. Results. A total of 10,421 people was surveyed. A $2.7 \%$ had cough with phlegm for $\geq 7$ days and only $1.1 \%$ were SR; 215 patients were screened for tuberculosis. Seven $(5.9 \%)$ of the RS and eight $(4.8 \%)$ of the patients with a $7-14$-day cough had tuberculosis. Conclusions. The percentage of RS in the Ministry of Health's health facilities in Lima was less than the expected $5 \%$, ranging from $0.8 \%$ in Callao to $1.5 \%$ in Lima City. The percentage of tuberculosis among coughers (lasting 7-14 days) and RS is consistent with the findings of other local studies. Consideration should be given to lowering the RS target to less than $5 \%$ and expanding tuberculosis screening to patients with cough $\geq 7$ days at the Health Divisions with high tuberculosis incidence.
\end{abstract}

Keywords: Screening; Tuberculosis; Health systems; Peru (source: MeSH NLM).

Médica infectóloga, $\mathrm{PhD}$ en Ciencias de la Salud; ${ }^{\mathrm{b}}$ médico epidemiólogo, doctor en Medicina; ${ }^{\mathrm{c}}$ médico cirujano, magíster en Epidemiología Clínica. Recibido: 15/01/2019 Aprobado: 15/05/2019 En línea: 28/06/2019 


\section{INTRODUCCIÓN}

Desde su creación en las décadas de 1970s y 1980s, los programas de control de tuberculosis han basado la identificación de casos nuevos de tuberculosis en la búsqueda pasiva de sintomáticos respiratorios (SR), definidos como pacientes con tos y expectoración por más de dos semanas entre aquellos que se atienden en la consulta médica de los establecimientos de salud. A estos pacientes se les pide dos muestras de esputo para realizar el despistaje de tuberculosis mediante la búsqueda de bacilos ácido-alcohol resistentes (BAAR) en el examen del extendido con coloración de Ziehl-Neelsen. Esta intervención está dirigida a impulsar la detección temprana y a su vez el tratamiento oportuno, que son pilares fundamentales para lograr disminuir la transmisión de la enfermedad ${ }^{(1,2)}$. El fundamento para esta intervención es que se ha observado una asociación significativa entre la búsqueda y examen de los SR y el número total de casos de tuberculosis detectados ${ }^{(3)}$.

Se estima que la prevalencia de SR estaría alrededor del $5 \%$ en pacientes mayores de 15 años que se atienden en consulta externa ${ }^{(4)}$. Este rango puede variar entre $3,5 \mathrm{y}$ $10,7 \%$ de acuerdo a algunos estimados en otros países ${ }^{(5-8)}$. También se sabe que este valor está influenciado por factores como la localización geográfica, el tipo de establecimiento de salud, la incidencia de enfermedades respiratorias (incluyendo tuberculosis) en la región, el motivo de consulta, y probablemente la estacionalidad ${ }^{(9)}$, entre otros.

En Perú no se han realizado estudios adecuadamente diseñados que permitan estimar la proporción de SR; sin embargo, es uno de los indicadores operacionales de detección de tuberculosis más importante, con una meta establecida en al menos $5 \%$ del total de atenciones. Este indicador se mide a través de la información programática, registrada en los reportes periódicos hechos por las Direcciones de Salud (DISAs) y Direcciones Regionales de Salud (DIRESAs), que luego son consolidados y publicados por la Estrategia Sanitaria de Prevención y Control de Tuberculosis (ESNPCTB) del Ministerio de Salud (MINSA). Según esta fuente de información, el promedio nacional de SR en el 2010 fue de $3 \%$, con un rango de 1,5\% hasta $5,8 \%{ }^{(10)}$ y de $3,3 \%$ en el $2016^{(11)}$. No se conocen las causas por las que no se logra llegar a la meta de $5 \%$, pero se postula que podría ser debido a la variabilidad en la carga de enfermedad, a inadecuadas actividades de detección y diagnóstico o a que la meta no concuerda con la realidad sanitaria peruana.

Desde otro punto de vista, con la finalidad de optimizar la detección de casos, algunos estudios indican que la definición de sospechoso podría ajustarse a tos por siete o más días en lugar de tos por más de 2 semanas. Esto contribuiría a una mayor captación de pacientes, aunque

\section{MENSAJES CLAVE}

Motivación para realizar el estudio. Los programas de control de tuberculosis siguen la recomendación de la Organización Panamericana de la Salud de llegar a un 5\% de tamizaje de tuberculosis entre los pacientes que acuden a consulta externa. Sin embargo, pocas veces se logra alcanzar esta cifra.

Principales hallazgos. Se hizo una encuesta para ver cuál es el porcentaje real de pacientes con tos por más de 14 días. Encontramos que dependiendo de la localización, alrededor de $1 \%$ de las personas tiene tos de dicha duración y no el 5\% que clásicamente se ha buscado.

Implicancias. Sería recomendable reevaluar si se debe disminuir la meta del 5\% de tamizaje de tuberculosis en consulta externa.

podría poner a los laboratorios en riesgo de sobrecarga ${ }^{(6,10)}$. En todo caso, constituye un elemento adicional por evaluar.

El objetivo de este estudio fue determinar el porcentaje de $\mathrm{SR}$, según la definición programática, entre las personas que en condiciones habituales acuden a consulta en los establecimientos de salud de primer nivel del MINSA en Lima, e identificar el porcentaje de pacientes con tuberculosis entre los SR y entre tosedores de más de siete días.

\section{MÉTODOS}

Se realizó un estudio transversal; se encuestó a personas de 15 años o más que acudieron a consulta externa en establecimientos de salud de primer nivel (centros de salud) en Lima y Callao pertenecientes al sistema de salud público (MINSA). El reclutamiento se llevó a cabo entre abril y setiembre de 2012. Se incluyeron 57 microrredes de salud, de cada una se eligió un centro de salud que fuera la cabeza de la microrred o que hubiera tenido el mayor número de atenciones en el 2011. En cinco microrredes con pocas atenciones se eligieron dos establecimientos de salud; $y$ cinco microrredes se excluyeron por inaccesibilidad geográfica.

\section{POBLACIÓN Y MUESTRA}

El tamaño muestral para una proporción estimada del $5 \%$ con un nivel de confianza del $95 \%$ y un margen de error del $1 \%$, tomando como total de atenciones las realizadas en Lima el 2009 (15 847 554) fue de 2401, el cual aplicamos para cada una de las DISAs y DIRESA incluidas. Considerando un $10 \%$ adicional de pérdidas, tuvimos una muestra total de 10564 .

\section{PROCEDIMIENTO}

La recolección de datos se realizó en dos etapas y estuvo a cargo de seis enfermero(a)s, capacitados para el estudio. 
En la primera etapa, los encuestadores invitaron a participar en el estudio a todo paciente mayor de 15 años que acudía a consulta externa, los cuales se encontraban esperando en la cola de atención. Se ofrecía participar a todos los consultantes de ese turno. A los pacientes que aceptaban participar se les aplicó un breve cuestionario mediante el cual se recogió información sobre edad, género (en caso de mujeres, si era gestante), consultorio al que acudía, presencia de tos, y en caso de tener tos, la duración y si ésta era el motivo de la consulta. Se excluyeron a las personas en tratamiento antituberculoso ya iniciado o que no pudieran responder las encuestas debido a discapacidad auditiva, enfermedad psiquiátrica u otras condiciones físicas por las cuales no pudieran brindar información.

A las personas que tenían tos y expectoración por siete o más días se les invitó a participar en la segunda etapa del estudio, para lo cual se les pidió firmar un consentimiento informado y responder un cuestionario acerca de datos sociodemográficos (procedencia, ocupación y grado de instrucción), antecedentes (patológicos y epidemiológicos) y sintomatología al momento de la encuesta (expectoración, fiebre, pérdida de peso, sudoración nocturna y hemoptisis). Adicionalmente, se les solicitó una muestra de esputo que debía ser recolectada en ese momento ("on the spot").

Las muestras de esputo fueron divididas en alícuotas para ser sometidas a los siguientes exámenes: baciloscopía con coloración de Ziehl-Neelsen directa de esputo y de esputo concentrado, cultivo en Lowenstein-Jensen y observación microscópica de susceptibilidad a drogas (MODS). Todos los exámenes microbiológicos fueron realizados en el laboratorio referencial de tuberculosis de la DIRESA Callao afiliado al Instituto de Medicina Tropical "Daniel A. Carrión". Si la muestra era insuficiente, se priorizó la baciloscopía directay cultivo en Lowenstein-Jensen. Se definió como caso de tuberculosis a todo paciente que presentó baciloscopía, cultivo en Lowenstein-Jensen o MODS positivo sin antecedente de diagnóstico de tuberculosis en los últimos seis meses. Todos los resultados fueron entregados a los responsables de tuberculosis de los establecimientos para hacer el seguimiento correspondiente.

\section{ANÁLISIS ESTADÍSTICO}

Se realizó el análisis descriptivo usando como medidas de resumen porcentajes para variables cualitativas, y media, desviación estándar, mediana e intervalo intercuartil para variables cuantitativas. Adicionalmente, se estableció como resultado la presencia de tuberculosis, de acuerdo a la definición planteada, y se evaluó la asociación con los factores demográficos y clínicos más relevantes. Se utilizaron las pruebas de chi cuadrado o Fisher para variables cualitativas, y la prueba de $\mathrm{t}$ de Student para variables cuantitativas. Un valor de p menor a 0,05 fue considerado significativo. Se utilizó el paquete estadístico SPSS Statistics for Windows, versión 17 (SPSS Inc., Chicago, III., USA).

\section{ASPECTOS ÉTICOS}

El presente estudio fue aprobado por el comité de ética del Instituto Nacional de Salud. Adicionalmente, se obtuvo aprobación de todas las DISAs participantes. Los mayores de edad firmaron un consentimiento informado; los menores de 18 años firmaron un asentimiento y uno de sus padres el consentimiento informado.

\section{RESULTADOS}

Accedieron a responder y completar la encuesta 10421 personas. La edad promedio de los pacientes incluidos fue 33 años (15-102 años). La Tabla 1 muestra las características demográficas y de tipo de consulta para cada una de las Direcciones de Salud de Lima y Callao. No hubo diferencias significativas en estas características entre estos grupos.

Del total de consultantes, 1561 (15\%) refirió haber tenido tos de cualquier duración, con una duración media de 7,4 días (1-1095 días). En el 31,7\% de estas personas, la tos fue el principal motivo de consulta.

Doscientos ochenta y tres $(2,7 \%)$ de los consultados refirió tener tos con expectoración por siete días o más y $118(1,1 \%)$ cumplieron con el criterio de SR de tos con expectoración por más de 14 días. Se observó que la DISA Lima Ciudad presentó una mayor proporción de SR (1,5\%), sin embargo, esta diferencia no fue estadísticamente significativa frente a la segunda DISA con mayor proporción que fue Lima Sur $(p=0,137)$.

En el análisis bivariado, los varones tuvieron mayor probabilidad que las mujeres de tener tos con expectoración por siete o más (OR 1,4; IC 95\%: 1,1-1,8; $p<0,01$ ), pero no de tener tos con expectoración por 14 o más días (OR 1,2; IC 95\%: 0,8-1,8; $p<0,01)$. Las personas con tos de siete a catorce días tuvieron mayor edad que los que no cumplían dicho criterio (42,6 $\pm 19,6$ vs. $36,8 \pm 17,4 ; p<0,001)$; la misma observación se realizó en el caso de SR (46,2 \pm 19,9 vs. $36,9 \pm 17,4 ; p<0,001)$. La Tabla 2 muestra el porcentaje de SR y de pacientes con tos por siete días o más.

De las 283 personas que tenían tos por siete o más días, 46 personas no accedieron a participar en la segunda fase, 15 no pudieron recolectar muestra en el momento y siete tuvieron muestra de esputo insuficiente, por lo que 215 fueron incluidas para despistaje de tuberculosis (Figura 1). Adicionalmente, nueve muestras fueron insuficientes para realizar la prueba MODS.

La edad promedio de este subgrupo fue 41,8 \pm 18,9 años (rango de 15 a 92 años), el $72 \%$ (160) fue de sexo femenino. El 8,6\% de SR refirió antecedente de tuberculosis hacía más de seis meses. Todos ellos reportaron haber terminado satisfactoriamente su tratamiento. Solo dos participantes 


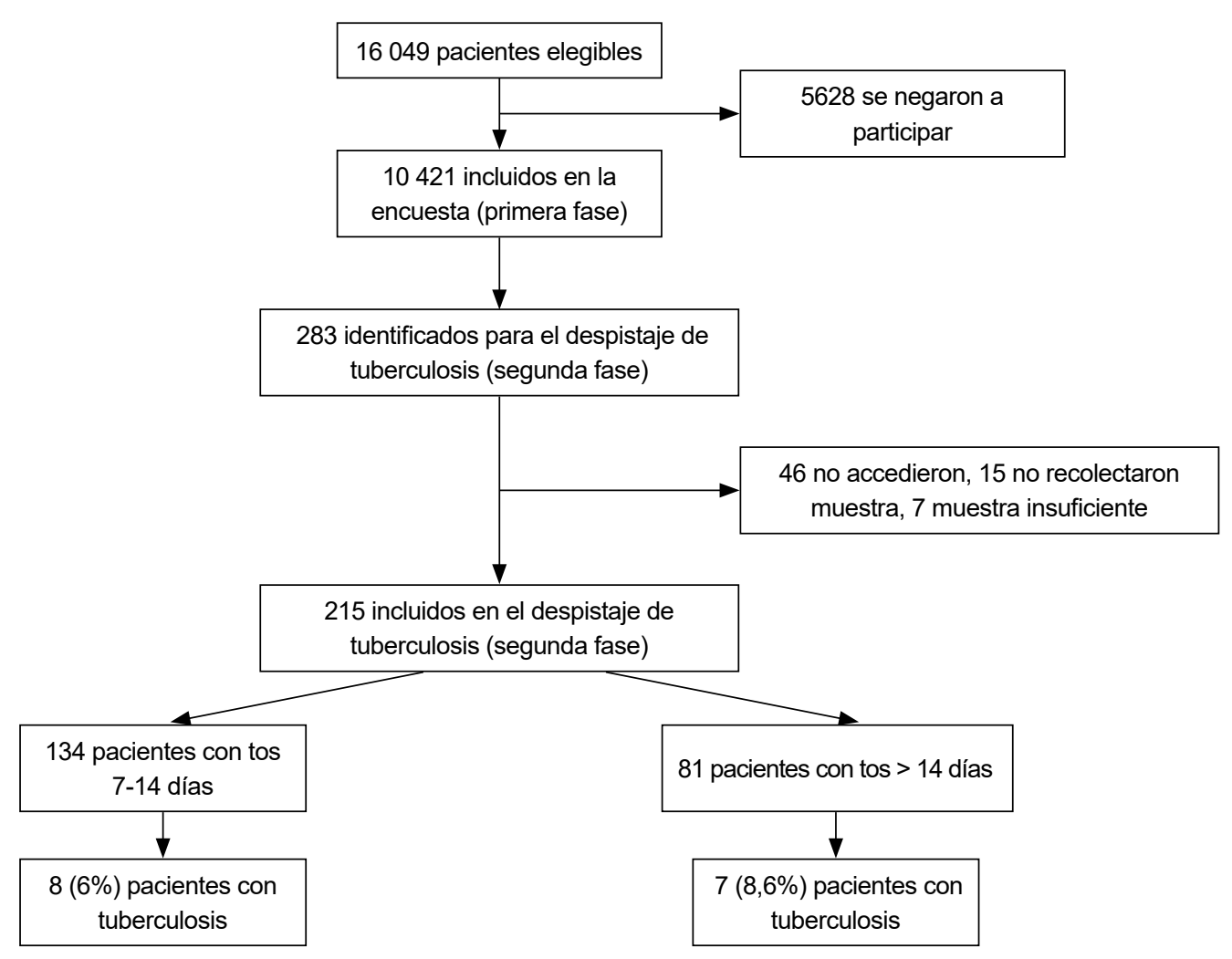

Figura 1. Flujograma de pacientes incluidos en el estudio (sintomáticos respiratorios y pacientes con tuberculosis), Lima y Callao, 2012

mencionaron vivir en la actualidad con una persona con diagnóstico de tuberculosis (contacto) y $44(19,9 \%)$ reportaron haber vivido anteriormente con una persona con diagnóstico de tuberculosis. Dos pacientes tenían diagnóstico de infección por el virus de la inmunodeficiencia humana $(\mathrm{VIH})$, ambos en tratamiento antirretroviral. El promedio de días con tos fue de 17,2 \pm 17,7 días (rango 7-120 días).

De acuerdo a los resultados de laboratorio, quince $(5,3 \%)$ de estos pacientes cumplieron con nuestra definición de tuberculosis. De estos, cinco tuvieron todas las pruebas diagnósticas positivas, dos tuvieron baciloscopía y cultivo/ MODS positivos, cuatro solo baciloscopía positiva y cuatro tuvieron baciloscopía negativa pero cultivo/MODS positivo (Tabla 3).

\section{DISCUSIÓN}

La ESNPCTB establece como indicador operacional de detecciones más importantes la proporción de SR

Tabla 1. Características demográficas y de atención de salud, según Dirección de Salud en Lima y Callao, 2012

\begin{tabular}{lcrrrr}
\hline Variable & $\begin{array}{c}\text { Lima Sur } \\
\mathbf{n}(\%)\end{array}$ & $\begin{array}{c}\text { Callao } \\
\mathbf{n}(\%)\end{array}$ & $\begin{array}{c}\text { Lima Este } \\
\mathbf{n}(\%)\end{array}$ & $\begin{array}{c}\text { Lima Ciudad } \\
\mathbf{n}(\%)\end{array}$ & $\begin{array}{c}\text { Total } \\
\mathbf{n}(\%)\end{array}$ \\
\hline Encuestados & $2655(25,5)$ & $2144(20,6)$ & $2606(25,0)$ & $3016(28,9)$ & $10421(100,0)$ \\
\hline Mediana de edad (RIQ) (años) & $33(24-53,5)$ & $37,5(25-54)$ & $32(22-50)$ & $30(22-53)$ & $33(23,5-52,5)$ \\
Mujeres & $1985(74,8)$ & $1693(79,0)$ & $2011(77,2)$ & $2352(78,0)$ & $8041(77,2)$ \\
Consultorio de Medicina & $1108(41,7)$ & $932(43,5)$ & $1002(38,5)$ & $1290(42,8)$ & $4332(41,6)$ \\
Consultorio de Obstetricia & $667(25,1)$ & $606(28,3)$ & $632(24,3)$ & $690(22,9)$ & $2595(24,9)$ \\
\hline Atención rápida & $208(7,8)$ & $129(6,0)$ & $203(7,8)$ & $144(4,8)$ & $683(6,6)$ \\
\hline Otros* & $672(25,3)$ & $477(22,2)$ & $769(29,5)$ & $892(29,6)$ & $2811(27,0)$ \\
\hline
\end{tabular}

* Incluye consultorios de cirugía, psicología, servicio social y nutrición $\mathrm{RIQ}$ : rango intercuartil 
Tabla 2. Porcentaje de pacientes con tos y expectoración de acuerdo a duración de los síntomas y Dirección de Salud en Lima y Callao, 2012

\begin{tabular}{|c|c|c|c|c|c|}
\hline Variable & $\begin{array}{c}\text { Lima Sur } \\
n(\%) \\
{[I C \quad 95 \%]}\end{array}$ & $\begin{array}{c}\text { Callao } \\
\text { n (\%) } \\
\text { [IC 95\%] }\end{array}$ & $\begin{array}{c}\text { Lima Este } \\
\text { n (\%) } \\
\text { [IC 95\%] }\end{array}$ & $\begin{array}{c}\text { Lima Ciudad } \\
\text { n (\%) } \\
\text { [IC 95\%] }\end{array}$ & $\begin{array}{c}\text { Total } \\
\mathbf{n}(\%) \\
\text { [IC 95\%] }\end{array}$ \\
\hline Encuestados & $2655(25,5)$ & $2144(20,6)$ & $2606(25,0)$ & $3016(28,9)$ & $10421(100,0)$ \\
\hline Tos por 7-14 días & $\begin{array}{r}45(1,7) \\
{[1,2-2,2]}\end{array}$ & $\begin{array}{c}18(0,8) \\
{[0,4-1,2]}\end{array}$ & $\begin{array}{r}30(1,2) \\
{[0,6-1,4]}\end{array}$ & $\begin{array}{r}72(2,4) \\
{[1,9-3,0]}\end{array}$ & $\begin{array}{r}165(1,6) \\
{[1,4-1,9]}\end{array}$ \\
\hline Tos por más de 14 días (SR) & $\begin{array}{r}31(1,2) \\
{[0,8-1,7]}\end{array}$ & $\begin{array}{r}17(0,8) \\
{[0,5-1,3]}\end{array}$ & $\begin{array}{c}26(1,0) \\
{[0,7-1,5]}\end{array}$ & $\begin{array}{r}44(1,5) \\
{[1,1-2,0]}\end{array}$ & $\begin{array}{l}118(1,1) \\
{[0,9-1,4]}\end{array}$ \\
\hline Tos por 7 días o más & $\begin{array}{r}76(2,9) \\
{[2,3-3,6]}\end{array}$ & $\begin{array}{r}35(1,6) \\
{[1,1-2,3]}\end{array}$ & $\begin{array}{r}56(2,1) \\
{[1,6-2,8]}\end{array}$ & $\begin{array}{c}116(3,8) \\
{[3,2-4,6]}\end{array}$ & $\begin{array}{r}283(2,7) \\
{[2,4-3,1]}\end{array}$ \\
\hline
\end{tabular}

SR: sintomáticos respiratorios

identificada entre las atenciones de los establecimientos de salud del MINSA en mayores de 15 años ${ }^{(12)}$. Este indicador es especialmente enfatizado en la evaluación de los establecimientos de salud e incluso es denominado «indicador trazador» ${ }^{(13)}$, considerándose que mide la intensidad de búsqueda de SR entre los pacientes atendidos. La evidencia para sustentar el porcentaje de $5 \%$ es sin embargo insuficiente. Más aún cuando recientemente la Contraloría General de la República ha hecho un llamado a cumplir con esta cifra ${ }^{(14)}$. Esto, desde un punto de vista administrativo puede resultar correcto, pero, como se muestra en este estudio, alcanzar esta meta ni corresponde a la realidad ni aseguraría un adecuado uso de los recursos en la captación de casos de tuberculosis.

El porcentaje de SR identificado en pacientes mayores de 15 años que acuden a consulta externa de los establecimientos de salud del primer nivel de Lima y Callao en nuestro estudio fue tan solo de 1,1\%, porcentaje significativamente menor al $5 \%$ esperado, con un rango de $0,8 \%$ en Callao hasta $1,5 \%$ en Lima Ciudad, y menor al reportado por las DIRESAs en sus informes operacionales (10). Estos reportes se acercan más al $3 \%$ hallado en un análisis secundario de la ENDES ${ }^{(15)}$. Ello podría deberse a que nuestro estudio, a diferencia de las actividades reportadas por las DIRESAs y el estudio de fuente secundaria, incluyó a una mayor proporción de establecimientos de salud que eran cabeceras de red, y estos por lo general son centros materno-infantiles, en los que la población consultante es más femenina y acude por motivos relacionados al embarazo o la atención del niño, lo que es menos frecuente en otro tipo de centros de salud.

Independientemente de esta diferencia, los hallazgos de nuestro estudio son consistentes con los reportes de las DISAs, con el estudio mencionado y otros estudios latinoamericanos ${ }^{(16,17)}$ que muestran que el $5 \%$ de meta establecida para SR no corresponde a lo encontrado en los establecimientos de salud y debe de ser reevaluado.

Establecer una meta mayor a la cantidad que realmente existe, puede generar una sobrecarga de baciloscopías, muchas innecesarias, y un empleo ineficiente de recursos. Esto podría explicar por qué la proporción de SR identificados reportada por el MINSA (3,4\%) es mucho mayor a lo obtenido en nuestro estudio, pero en cambio, el porcentaje de baciloscopías positivas $(1,6 \%)$ entre SR es menor al 5,9\% encontrado en este estudio ${ }^{(13)}$.

Las consecuencias de la sobrestimación del número de SR son importantes para el sistema de salud. El número de SR esperados en el Perú es de más de dos millones, lo cual implicaría hacer cuatro millones de baciloscopías. En el 2012, se identificaron 1,4 millones de SR, en quienes se realizaron 2,4 millones de baciloscopías, identificando poco más de 13000 nuevos casos de tuberculosis pulmonar con baciloscopía positiva (cinco casos por cada 1000 baciloscopías). El alto número de baciloscopías realizadas con un rendimiento diagnóstico tan bajo implica

Tabla 3. Porcentaje de pacientes con tuberculosis entre sintomáticos respiratorios y personas con tos por más de 7 días, según Dirección de Salud en Lima y Callao, 2012

\begin{tabular}{|c|c|c|c|c|c|}
\hline & $\begin{array}{l}\text { Lima Sur } \\
\text { n (\%) }\end{array}$ & $\begin{array}{l}\text { Callao } \\
\text { n (\%) }\end{array}$ & $\begin{array}{l}\text { Lima Este } \\
\text { n (\%) }\end{array}$ & $\begin{array}{l}\text { Lima Ciudad } \\
\text { n (\%) }\end{array}$ & $\begin{array}{l}\text { Total } \\
\text { n (\%) }\end{array}$ \\
\hline Total de incluidos & $58(27,0)$ & $28(13,0)$ & $43(20,0)$ & $86(40,0)$ & $215(100,0)$ \\
\hline Tuberculosis en personas con tos por 7-14 días & $0 / 34(0,0)$ & $2 / 17(11,8)$ & $4 / 26(15,4)$ & $2 / 56(3,6)$ & $8 / 133(6,0)$ \\
\hline $\begin{array}{l}\text { Tuberculosis en personas con tos por más de } 14 \\
\text { días (SR) }\end{array}$ & $1 / 22(4,5)$ & $0 / 12(0,0)$ & $3 / 17(17,6)$ & $3 / 31(9,7)$ & $7 / 82(8,5)$ \\
\hline Tuberculosis en personas con tos de 7 a más días & $1 / 58(1,7)$ & $2 / 28(7,1)$ & $7 / 43(16,3)$ & $5 / 86(5,8)$ & $15 / 215(7,0)$ \\
\hline
\end{tabular}

SR: sintomáticos respiratorios 
una innecesaria carga de trabajo para el personal de salud y los laboratorios. Probablemente, una mejor estrategia sería focalizar los esfuerzos de detección en poblaciones con mayor riesgo desde el punto de vista geográfico (en zonas de alto riesgo de transmisión) y epidemiológico (en poblaciones más expuestas como los contactos), así como mejorar la aproximación del personal de salud a pacientes con sospecha de tuberculosis ${ }^{(18)}$.

Otro de los resultados resaltantes es el alto porcentaje $(5,8 \%)$ de casos de tuberculosis encontrado entre pacientes con tos entre 7-14 días, próximo al de aquellos con tos por más de 14 días (8,3\%). Nuestros datos son consistentes con los resultados obtenidos por Otero y colaboradores, quienes encontraron $5 \%$ de casos de tuberculosis en pacientes con tos de $7-14$ días y $10 \%$ en pacientes con tos de más de 14 días ${ }^{(19)}$. En este sentido, sería interesante realizar más estudios para determinar qué poblaciones se podrían beneficiar de disminuir el criterio de días de tos a siete para la realización de baciloscopías a fin de lograr captar a más pacientes, en particular en escenarios de alta incidencia. En nuestro estudio, se hubieran perdido casi la mitad de casos de tuberculosis de no haber incluido a aquellos pacientes con tos entre 7-14 días. Paradójicamente, ello podría implicar aumentar aún más el excesivo número de baciloscopías de diagnóstico; por eso es que se tendría que focalizar esta intervención únicamente a población de alto riesgo.

Cabe decir que en este estudio, la baciloscopía tuvo un rendimiento diagnóstico similar al del cultivo en Lowenstein-Jensen y MODS, sin embargo, hubo varias muestras insuficientes para realizar el MODS, por lo cual las contribuciones de ambas pruebas no son comparables. Consideramos que si bien la baciloscopía sigue siendo una herramienta importante para el diagnóstico de tuberculosis, de igual manera se debe definir el rol de los cultivos, y en la actualidad, de las pruebas moleculares en el diagnóstico de caso ${ }^{(20)}$

Este estudio presenta asimismo algunas limitaciones. En primer lugar, se trata de una estimación puntual, que podría no ser representativa de lo que acontece durante todo el año. Sin embargo, el estudio fue realizado en los meses de marzo a septiembre, que corresponden al otoño e invierno peruanos, por lo que, en todo caso, nuestro porcentaje de detección de SR podría ser algo mayor al promedio anual. Por otro lado, el porcentaje de rechazo a la encuesta fue alto. Solo se realizó una muestra de esputo, lo cual podría subestimar el número de casos de tuberculosis, aunque no el porcentaje de SR. Ello significaría que las diferencias entre la prevalencia de tuberculosis observada entre SR y las reportadas por el MINSA podrían ser aún mayores. Por último, esta información fue recolectada en el 2012, sin embargo, consideramos que no existen elementos para hacernos pensar que la situación haya cambiado de manera significativa desde entonces.

Tomada en conjunto, esta información nos indica que si los esfuerzos se centran en captar al paciente con síntomas de tos y expectoración para la realización de la baciloscopía y no en lograr alcanzar una meta obligatoria, se podría contar con un despistaje más eficiente ${ }^{(21-24)}$. Nuestro estudio es el primer estudio en Lima y Callao diseñado específicamente para evaluar el porcentaje de SR en los establecimientos de salud públicos sobre una muestra de más de 10000 participantes. Por lo mismo, consideramos que los hallazgos son relevantes y pueden contribuir a informar la toma de decisiones en este tema.

En conclusión, consideramos que la meta de 5\% de SR como indicador operacional de detección debe ser reevaluada de manera urgente, pues el esfuerzo por alcanzarla puede generar un uso masivo de baciloscopías de manera ineficiente. Es necesario diseñar y evaluar en estudios operativos mejores indicadores de detección que permitan un uso eficiente de los limitados recursos disponibles. Por otro lado, consideramos importante evaluar, idealmente a través de ensayos clínicos u otros estudios de intervención, el impacto de realizar despistaje de tuberculosis en casos de tos con más de siete días en poblaciones de alto riesgo de tuberculosis.

Contribuciones de los autores: LS participó en la concepción del estudio, la interpretación de datos, la redacción del manuscrito y la aprobación definitiva de la versión a publicar. JOA participó en el diseño del estudio, la interpretación de los datos, la redacción del manuscrito y la aprobación definitiva de la versión a publicar. JP participó en el diseño del estudio, la adquisición de los datos para el trabajo; la interpretación de los datos, la redacción del manuscrito y la aprobación definitiva de la versión a publicar. RT participó en la adquisición de los datos para el trabajo; la redacción del manuscrito y la aprobación definitiva de la versión a publicar. Todos los autores se hacen responsables del contenido del artículo.

Fuentes de financiamiento: El estudio fue financiado en su integridad por el Instituto Nacional de Salud del Perú.

Conflictos de interés: Todos los autores declaran que no tienen conflicto de interés alguno.

\section{REFERENCIAS BIBLIOGRÁFICAS}

1. Organización Mundial de la Salud. 10 datos sobre la tuberculosis [Internet]. WHO; 2017 [citado el 17 de mayo de 2019]. Disponible en: http://www.who. int/features/factfiles/tuberculosis/es/ index.html

2. Ministerio de Salud. Situación actual del control de la Tuberculosis [Internet].
Lima: MINSA; 2006 [citado el 17 de mayo de 2019]. Disponible en http:// bvs.minsa.gob.pe/local/dgsp/145_ construy2.pdf 
3. Perú, Ministerio de Salud. Resolución Ministerial 023-2010-SA. Plan Estratégico Multisectorial contra la Tuberculosis. Lima: MINSA; 2010.

4. Bonilla C. Situación de la tuberculosis en el Perú: current status. Acta méd. peruana. 2008;25(3):163-70.

5. Bastos LG, Fonseca LS, Mello FC, Ruffino-Netto A, Golub JE, Conde MB. Prevalence of pulmonary tuberculosis among respiratory symptomatic subjects in an out-patient primary health unit. Int J Tuberc Lung Dis. 2007;11(2):156-60.

6. Ngadaya ES, Mfinanga GS, Wandwalo ER, Morkve O. Detection of pulmonary tuberculosis among patients with cough attending outpatient departments in Dar Es Salaam, Tanzania: does duration of cough matter?. BMCHealth Serv Res. 2009;9:112. doi: 10.1186/1472-6963-9-112

7. Henao-RiverosS, Sierra-Parada C,SánchezMorales E, Rodríguez A. Búsqueda de Tuberculosis en Pacientes Sintomáticos Respiratorios en Cuatro Hospitales de Bogotá D.C. Rev. Salud pública. 2007;9(3):408-19.

8. Arenas N, Torres E, Durango C, Cuervo L, Coronado S, Gómez A. Búsqueda Activa de Individuos con Tuberculosis Pulmonar y Extrapulmonar en CalarcáQuindío, Colombia, 2005. Rev. salud pública. 2008;10(2):279-89.

9. Behera D, Sharma PP. A retrospective study of seasonal variation in the number of cases diagnosed at a tertiary care tuberculosis hospital. Indian J Chest Dis Allied Sci. 2011;53(3):145-52.

10. Ministerio de Salud. Estrategia Sanitaria Nacional de Prevención y Control de Tuberculosis [Internet]. Lima: MINSA; 2010 [citado el 17 de mayo de 2019]. Disponible en http://www. minsa.gob.pe/pvigia/publicaciones/ tuberculosis/8Norma_TB2007.pdf

11. Ministerio de Salud. Situación de tuberculosis en el Perú [Internet]. Lima: MINSA; 2017 [citado el 17 de mayo de 2019]. Disponible en http://www. tuberculosis.minsa.gob.pe/portaldpctb/ recursos/20180605122521.pdf
12. Ministerio de Salud. Norma técnica de salud para la atención integral de las personas afectadas por tuberculosis [Internet]. Lima: MINSA; 2013 [citado el 17 de mayo de 2019]. Disponible en: http://www.tuberculosis.minsa.gob.pe/ portaldpctb/recursos/20190404120230. PDF

13. Ministerio de Salud. Estrategia sanitaria nacional de tuberculosis, Sala situacional [Internet]. Lima MINSA; 2013 [citado el 17 de mayo de 2019]. Disponible en: http://www.tuberculosis.minsa.gob.pe/

14. Perú, Contraloría General de la República, Departamento de auditoría de desempeño y medio ambiente. Informe No. 759-2017-CG/DEMA. Auditoría de desempeño a los servicios de diagnóstico, tratamiento y prevención secundaria de tuberculosis prestados por el Ministerio de Salud en Lima Metropolitana [Internet]. Lima: CGR; 2017 [citado el 17 de mayo de 2019]. Disponible en: http://lfweb.contraloria. gob.pe/BuscadorInformes / 0 / edoc/4245518/Informe_Control_7592017-CG-DEMA.pdf

15. Gutiérrez C, Roque J, Romaní F, Zagaceta J. Prevalencia de sintomáticos respiratorios en población peruana de 15 a más años: análisis secundario de la Encuesta Demográfica y de Salud Familiar, 2013 - 2015. Rev Peru Med Exp Salud Publica. 2017;34(1):98-104.

16. Daza Arana JE, Cubides Munevar AM, Lozada Ramos H. Prevalencia de sintomáticos respiratorios y factores relacionados en dos territorios vulnerables de Santiago de Cali. Hacia promoc. salud. 2016;21(1):63-76. doi: 10.17151/hpsal.2016.21.1.5

17. Moreira CMM, Zandonade E, Lacerda T, Maciel ELN. Sintomáticos respiratórios nas unidades de atenção primária no Município de Vitória, Espírito Santo, Brasil. Cadernos de Saúde Pública. 2010;26:1619-26.

18. Lin C-H, Tsai C-H, Liu C-E, Huang M-L, Chang S-C, Wen J-H, et al. "Cough officer screening" improves detection of pulmonary tuberculosis in hospital in-patients. BMC Public Health. 2010;10(1):238..

19. Otero L, Ugaz R, Dieltiens G, González E, Verdonck K, Seas C, et al. Duration of cough, TB suspects' characteristics and service factors determine the yield of smear microscopy. Trop Med Int Health. 2010;15(12):1475-80.

20. Frieden TR, Sterling TR, Munsiff SS, Watt CJ, Dye C. Tuberculosis. Lancet. 2003;362 (9387):887-99.

21. Romani F, Roque Henríquez J, Catacora López F, Hilasaca Yngas G. Conocimientos, percepciones y prácticas de personal de salud en la detección de sintomáticos respiratorios en una región de muy alto riesgo de trasmisión de tuberculosis en el Perú. An. Fac. med. 2016;77(2):123-7.

22. English RG, Bachmann MO, Bateman ED, Zwarenstein MF, Fairall LR, Bheekie A, et al. Diagnostic accuracy of an integrated respiratory guideline in identifying patients with respiratory symptoms requiring screening for pulmonary tuberculosis: a cross-sectional study. BMC Pulm Med. 2006;6:22.

23. Lainez YB, Todd CS, Ahmadzai A, Doocy SC, Burnham G. Prevalence of respiratory symptoms and cases suspicious for tuberculosis among public health clinic patients in Afghanistan, 2005-2006: Perspectives on recognition and referral of tuberculosis cases. Trop Med Int Health. 2009; 14(5):564-70.

24. Reis SP, Harter J, Lima LM, Vieira DA, Palha PF, Gonzales RI. Geographical and organizational aspects of primary health care services in detecting tuberculosis cases in Pelotas, Rio Grande do Sul, Brazil, 2012. Epidemiol Serv Saude. 2017;26(1):141-148.

\author{
Correspondencia: Lely Solari \\ Instituto Nacional de Salud. Lima, Perú. \\ Dirección: Capac Yupanqui 1400 - Jesús \\ María, Lima, Perú. \\ Teléfono: 999128241 \\ Email:lelysol@hotmail.com
}

